\title{
Search for charged long-lived heavy particles with the ATLAS experiment at the LHC
}

\author{
Elisa GUIDO* \\ On behalf of the ATLAS Collaboration \\ INFN Genova \\ E-mail: elisa.guido@ge.infn.it
}

\begin{abstract}
We report on a search for charged long-lived heavy particles, as predicted by several theories beyond the Standard Model. Such particles can be detected by the ATLAS experiment at the LHC through anomalous specific ionization measured in the Pixel detector, through their slow motion $(\beta<1)$ measured in the Calorimeters, and also through their possible muon-likeness in the Muon Spectrometer. Results of this search on the full data sample collected by ATLAS during 2011, corresponding to an integrated luminosity of $\sim 4.7 \mathrm{fb}^{-1}$, are shown, combining the information obtained from various sub-detectors.
\end{abstract}

36th International Conference on High Energy Physics,

July 4-11, 2012

Melbourne, Australia

\footnotetext{
* Speaker.
} 


\section{Introduction}

Heavy long-lived particles (LLPs) are predicted by several extensions of the Standard Model (SM). Some Supersymmetry (SUSY) models [1] predict the existence of meta-stable sleptons, in particular in Gauge-Mediated SUSY Breaking (GMSB) with the $\tau$ slepton $(\tilde{\tau})$ as the LLP, and of colored meta-stable squarks ( $(\tilde{q})$ and gluinos $(\tilde{g})$ in split SUSY. Squarks and gluinos can hadronize with either a light SM quark system or a gluon, forming a $R$-hadron. $R$-hadrons may be singly charged, doubly charged or neutral, they can either change their electric charge by nuclear scattering processes with the detector material, or decay in the detector given their finite lifetime.

Heavy LLPs should be produced at the LHC as massive particles. They are expected to move slowly $(\beta<1)$, thereby having a measurable time-of-flight, and to release an anomalous amount of energy while passing through the detector. They may also interact like heavy muons.

According to these signatures, several possible searches for LLPs [2] are performed at ATLAS [3], and described in this paper: an Inner Detector (ID)-only search, potentially sensitive to meta-stable $R$-hadrons not reaching farther sub-detectors, or to stable $R$-hadrons becoming neutral; an ID+Calorimeters ( $\mu$-agnostic) search, where the $R$-hadron is not required to reach the Muon Spectrometer (MS); a full-detector search, mainly intended for stable $R$-hadrons; and an ID+MS search, focusing on the search for sleptons.

\section{Data and Monte Carlo simulated samples}

The search presented here is based on $\sim 4.7 \mathrm{fb}^{-1}$ of $p p$-collision data collected in 2011. Monte Carlo (MC) simulated signal samples, re-weighted for the distribution of pile-up events to match the running conditions of the 2011 data, are used for efficiency studies and for setting limits.

The GMSB samples are generated with: the number of super-multiplets in the messenger sector equal to 3 , the messenger mass scale of $250 \mathrm{TeV}$, the sign of the Higgsino mass parameter equal to 1 , and the scale factor for the gravitino mass set at 5000. The ratio of the two Higgs doublets vacuum expectation values $(\tan \beta)$ varies between 5 and 40, the SUSY particle mass scale $(\Lambda)$ ranges from 50 to $150 \mathrm{TeV}$ and the corresponding $\tilde{\tau}$ masses from 122.2 to $465 \mathrm{GeV}$. The mass spectra are obtained from the SPICE program [4] and the events are generated using HERWIG [5].

The $R$-hadron samples are generated with $\tilde{g}(\tilde{q})$ masses from 300 to $1500 \mathrm{GeV}$ (200 to 1000 $\mathrm{GeV}$ ). The pair production of gluinos and squarks is simulated by PYTHIA [6]. Gluinos are assumed to hadronize with a gluon in $10 \%$ of the events. The simulation of $R$-hadron interactions in matter is handled by GEANT4 [7] and based on a generic model [8].

\section{Mass and $\beta$ measurements}

The masses of slow charged particles can be measured by relying on ID information only. In particular, the Pixel specific energy loss $(\mathrm{d} E / \mathrm{d} x)$ and the momentum measurements can be fitted to an empirical Bethe-Bloch function, thus deducing the relativistic boost factor of the particle. The measurable $\beta \gamma$ ranges between 0.2 and 1.5. This particle identification method [9] is based on a five-parameter function which describes the behavior of the most probable value of $\mathrm{d} E / \mathrm{d} x$ with $\beta \gamma$ :

$$
\mathscr{M}_{\frac{\mathrm{d} E}{\mathrm{~d} x}}(\beta \gamma)=\frac{p_{1}}{\beta^{p_{3}}} \ln \left(1+\left(p_{2} \beta \gamma\right)^{p_{5}}\right)-p_{4} .
$$



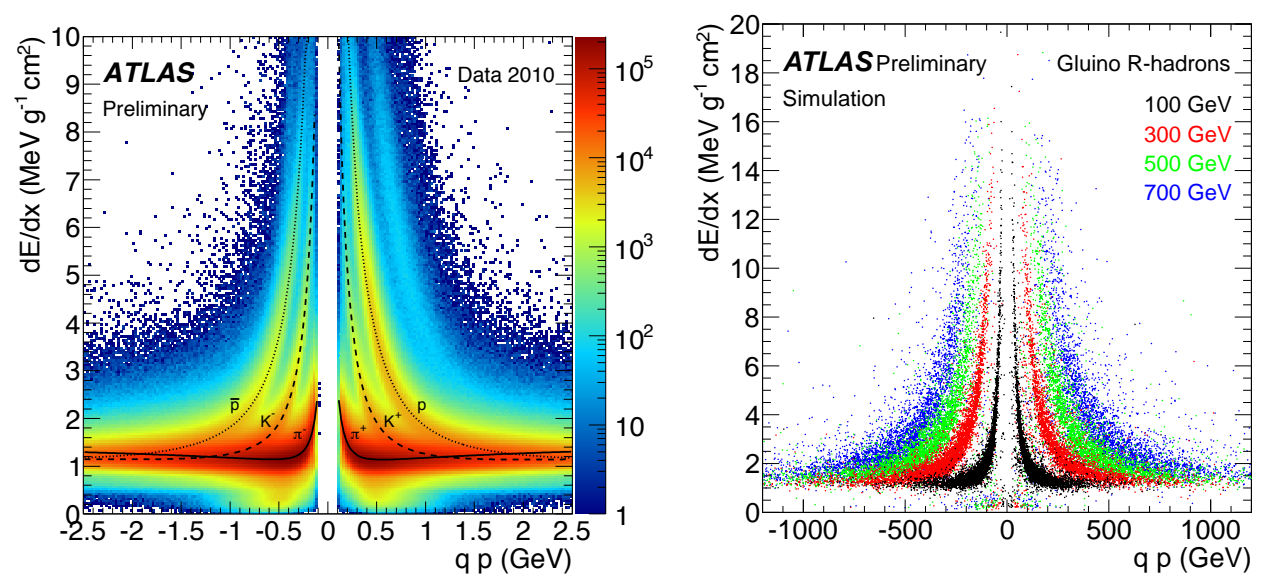

Figure 1: (left) Distribution of $\mathrm{d} E / \mathrm{d} x$ versus signed momentum for minimum bias 2010 collisions, with tracks reconstructed down to a $p_{T}$ of $100 \mathrm{MeV}$. The distributions of the most probable value for the fitted probability density functions of pions (solid), kaons (dashed) and protons (dotted) are superimposed [2]. (right) Simulated distributions of $\mathrm{d} E / \mathrm{d} x$ versus signed momentum for $R$-hadrons of various masses [2].

This function for known SM minimum ionizing particles (MIPs), superimposed on data for low momentum tracks, is shown in Fig. 1 (left). LLPs are expected to release more energy than MIPs, even for high momentum tracks, as can be seen in Fig. 1 (right), for singly charged $R$-hadrons of masses 100, 300, 500 and $700 \mathrm{GeV}$.

Both Tile and Liquid Argon Calorimeters can provide a good timing resolution, thus being able to distinguish between highly relativistic particles and slow LLPs. The MS can perform a $\beta$ measurement mainly thanks to the monitored drift-tube and resistive plate chambers; for slow LLPs a combined track re-fit including ID and MS information is used. For each track, the $\beta$ measurements from different detectors, if internally consistent, are also combined in a weighted average, the weights being obtained from the calculated error of each measurement, corrected for differences between data and MC for muons from $Z \rightarrow \mu \mu$ decays. The typical resolutions for Calorimeter-based and combined $\beta$ measurements are shown in Fig. 2.

\section{Event selection and background estimation}

The event selection is optimized separately for the different searches.

The slepton search requires a single muon trigger, which has a typical efficiency of $\sim 85 \%$. A loose selection is applied while looking for two LLP candidates, and identifies tracks with $p_{T}>50$ $\mathrm{GeV}$, with a veto for the invariant mass region between $\pm 10 \mathrm{GeV}$ with respect to the nominal $Z$ mass value, and a combined $\beta<0.95$; a tighter selection is instead applied when requiring only one LLP candidate, and it consists in asking for a track with $p_{T}>70 \mathrm{GeV}$, a $\beta$ measurement compatible at a $2 \sigma$ level among at least two different sub-detectors, and a cut on $m_{\beta}=p / \beta \gamma$, depending on slepton mass. The selection efficiency on the signal is typically of $\sim 20 \%$.

The $R$-hadron searches are based on a missing transverse energy $\left(E_{T}^{\text {miss }}\right)$ trigger, with a 60-70 $\mathrm{GeV}$ threshold and a typical efficiency of $\sim 15 \%$. For the full-detector and $\mu$-agnostic analyses, we look for a track of momentum between $140 \mathrm{GeV}$ and $3.5 \mathrm{TeV}$, and isolated from jets and other 

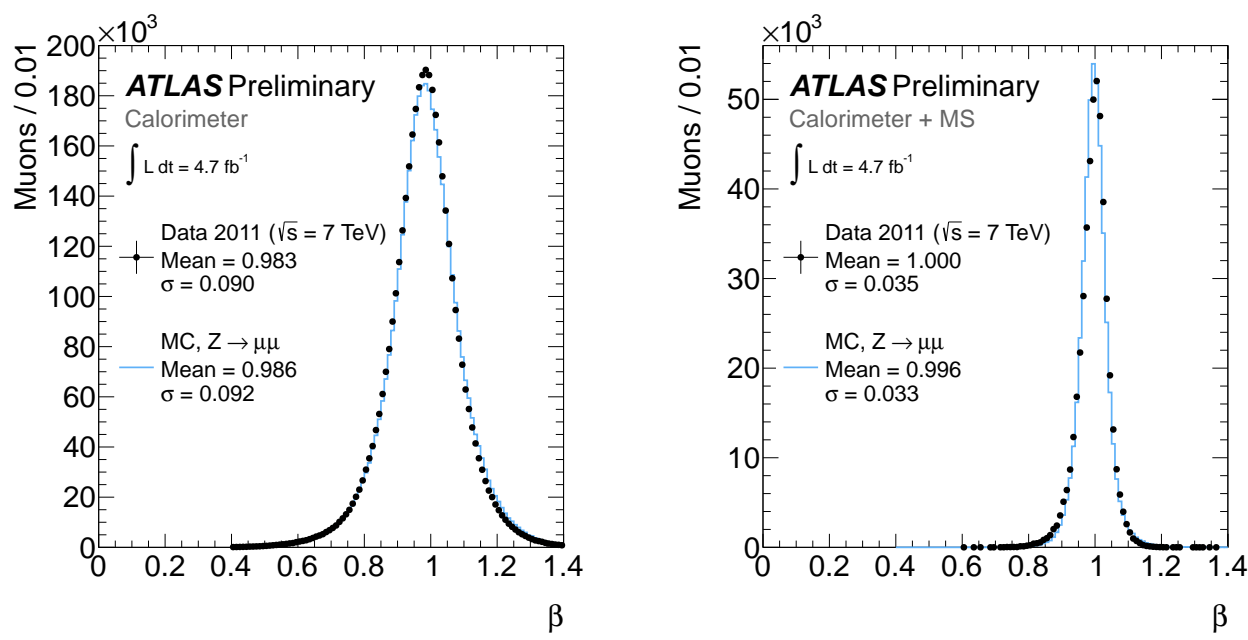

Figure 2: Distribution of $\beta$ (left) for the calorimeter and (right) for the combined resistive plate chambers, monitored drift-tube chambers and calorimeter measurements, obtained for selected $Z \rightarrow \mu \mu$ decays in data and MC simulation [2].

tracks, with a $\beta \gamma<1.5-2.0$ and either a combined or a calorimetric $\beta<0.8-0.9$. A tighter selection is applied in the case of the ID-only analysis: we search for events with $E_{T}^{\text {miss }}$ confirmed offline as greater than $85 \mathrm{GeV}$, and with a track of $p_{T}>50 \mathrm{GeV}$ and $p>100 \mathrm{GeV}$, more severely isolated, not compatible with the electron hypothesis, and with $\mathrm{d} E / \mathrm{d} x>(1.8+f(\eta)) \mathrm{MeVg}^{-1} \mathrm{~cm}^{2}$, where $f(\eta)$ accounts for a residual dependence of the ionization on the pseudorapidity. The selection efficiency on the signal varies with the $R$-hadron mass, and is typically of $\sim 11 \%(\sim 6 \%)$ for $m(\tilde{g})=900 \mathrm{GeV}$ in the full-detector (ID-only) search.

After the selection is applied, the background is mainly due to high- $p_{T}$ muons with mismeasured $\beta$. A data-driven approach is used to build high-statistics random samples of background events, starting from kinematic distributions obtained from data control samples.

\section{Systematic uncertainties}

Several systematic uncertainties affect this search. They can be grouped into uncertainties on the theoretical cross-sections, on the background estimation, on the luminosity, and on the signal efficiency. The latter are mainly due to systematic discrepancies between data and simulation in trigger efficiency, momentum resolution, $\beta$ and $\mathrm{d} E / \mathrm{d} x$ calibrations, and $E_{T}^{\text {miss }}$ scale. A summary of the uncertainties for the different analyses is given in Table 1.

\begin{tabular}{|l|c|c|}
\hline Systematic uncertainty & sleptons & R-hadrons \\
\hline Theoretical cross-sections & $5.0 \%$ & $15-30 \%$ \\
Signal efficiency & $4.4-6.3 \%$ & $11-13 \%$ \\
Background estimation & $11-13 \%$ & $15-20 \%$ \\
Luminosity & $3.9 \%$ & $3.9 \%$ \\
\hline
\end{tabular}

Table 1: Summary of systematic uncertainties, as in [2]. 

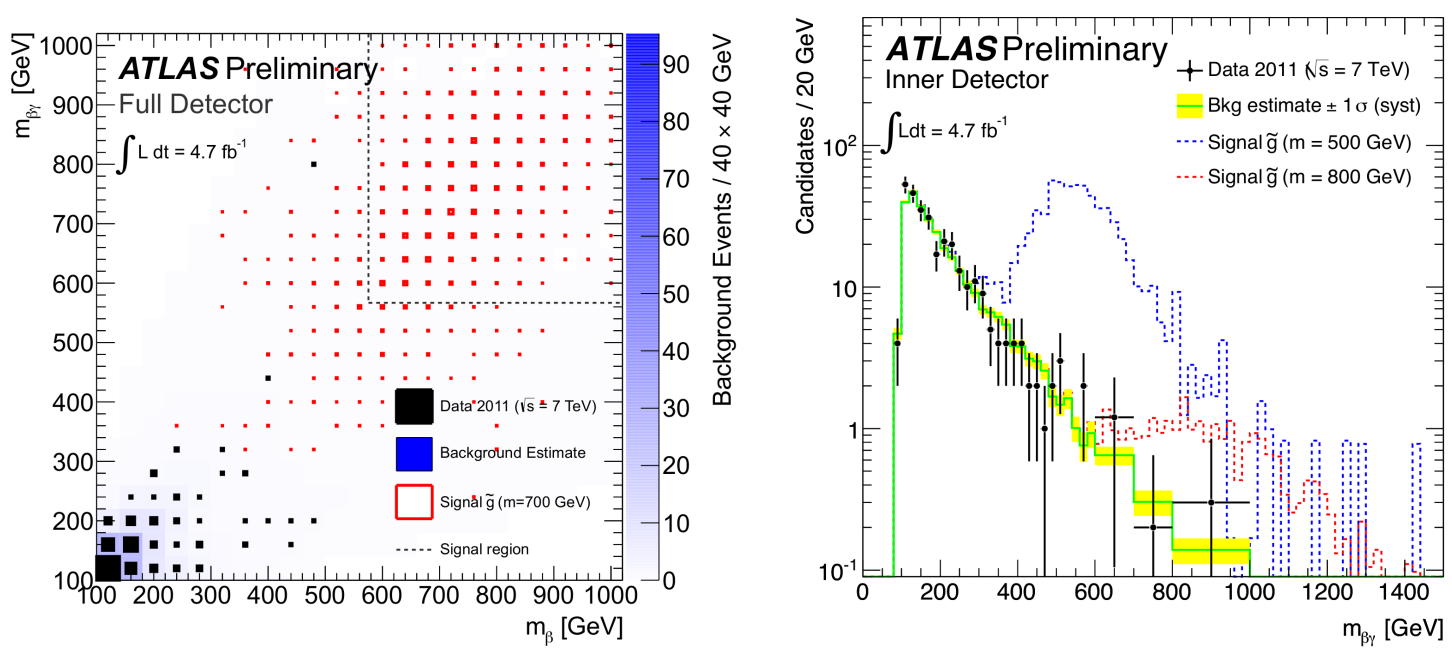

Figure 3: Observed data, background estimate and expected signal (left) in the full detector and (right) in the ID-only $R$-hadron search [2].

\section{Results}

Examples of the mass distributions for the expected signal and for the estimated background, while considering also systematic uncertainties, compared to the observed distributions on data, are shown in Fig. 3. Similar behaviors are found in the $\mu$-agnostic and slepton searches [2]. No significant indications of a signal above the background expectations are found, and $95 \%$ confidence level (CL) upper limits are set on the cross-section for the production of sleptons and $R$-hadrons, following the $C L_{s}$ prescriptions [10]. Lower limits on the masses for the LLPs can be deduced, as summarized in Table 2. In the GMSB scenario, limits are also set as a function of $\Lambda$ and $\tan \beta$. Two examples of the results are shown in Fig. 4.

\begin{tabular}{|l|c|c|c|}
\hline & full-detector & $\mu$-agnostic & ID-only \\
\hline $\mathrm{m}(\tilde{g})>[\mathrm{GeV}]$ & 985 & 989 & 940 \\
$\mathrm{~m}(\tilde{t})>[\mathrm{GeV}]$ & 683 & 657 & 604 \\
$\mathrm{~m}(\tilde{b})>[\mathrm{GeV}]$ & 612 & 618 & 576 \\
\hline \hline & \multicolumn{3}{|c|}{ sleptons } \\
\hline $\mathrm{m}(\mathrm{GMSB} \tilde{\tau})>[\mathrm{GeV}]$ & \multicolumn{2}{|c|}{$2700(5<\tan \beta<40)$} \\
$\mathrm{m}($ directly produced sleptons $)>[\mathrm{GeV}]$ & \multicolumn{2}{|c|}{$268<40)$} \\
\hline
\end{tabular}

Table 2: Summary of resulting 95\% CL limits on the masses of LLPs, as in [2].

\section{Conclusions}

A search for LLPs using data from $p p$-collisions collected by the ATLAS detector in 2011 has been presented. It represents an interesting complement to searches where SUSY particles decay promptly. Several signatures have been considered, and different detection techniques have 

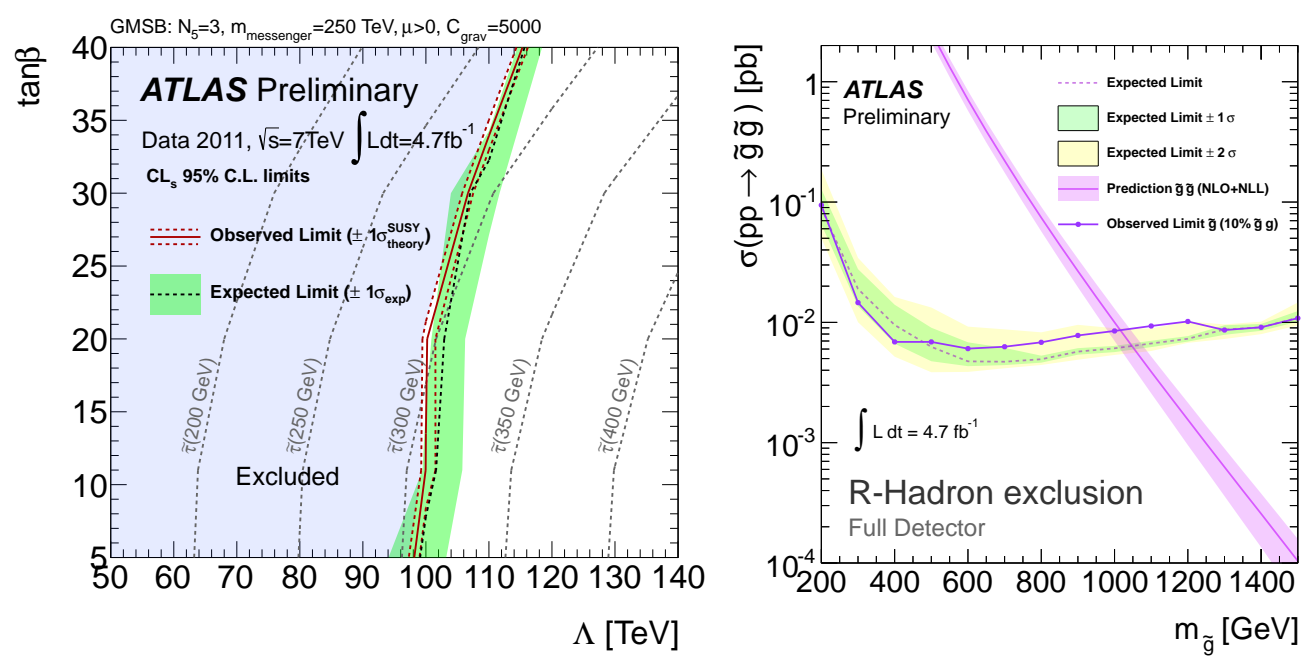

Figure 4: (left) Limits on $\Lambda$ and $\tan \beta$ in GMSB models. The expected limit is drawn as a dashed black line with green $1 \sigma$ band while the observed limit is a solid red line with the $1 \sigma$ theoretical uncertainty bounded by red dashed lines. (right) The cross-section exclusion limits for gluino $R$-hadrons using the full detector reconstruction are shown as the expected limit (dashed line) with \pm 1 (green) and $\pm 2 \sigma$ (yellow) uncertainty bands. The observed upper limit is represented by the solid line with markers. The purple solid line is the theoretical prediction for the production cross-section calculated at NLO+NLL [2].

been set up in order to perform this analysis. No significant deviations from the SM background expectations are found. The 95\% CL limits obtained on the cross-section for the production of LLPs, and therefore on the LLPs masses, extend ATLAS previous limits [11, 12], thanks both to the increased luminosity and to a more refined analysis approach.

\section{References}

[1] M. Fairbairn et al., Phys. Rept. 438 (2007) 1.

[2] ATLAS Collaboration, ATLAS-CONF-2012-075 (2012), https://cdsweb.cern.ch/record/1460272 .

[3] ATLAS Collaboration, JINST 3 (2008) S08003.

[4] G. Engelhard et al., arXiv:0904.1415 [hep-ph] (2009).

[5] G. Corcella et al., JHEP 0101 (2001) 010.

[6] T. Sjostrand, S. Mrenna, and P. Skands, JHEP 0605 (2006) 026.

[7] GEANT4 Collaboration, Nucl. Instrum. Meth. A 506 (2003) 250.

[8] R. Mackeprang and D. Milstead, arXiv:0908.1868 [hep-ph] (2009).

[9] ATLAS Collaboration, ATLAS-CONF-2011-016 (2011), https://cdsweb.cern.ch/record/1336519.

[10] A. L. Read, J. Phys. G 28 (2002) 2693.

[11] ATLAS Collaboration, Phys. Lett. B 701 (2011) 1.

[12] ATLAS Collaboration, Phys. Lett. B 703 (2011) 428. 